\title{
Mitochondrial Genome of Garcinia mangostana L. variety Mesta
}

Ching-Ching Wee ${ }^{1,2}$, Nor Azlan Nor Muhammad ${ }^{1}$, Vijay Kumar Subbiah ${ }^{2}$, Masanori Arita ${ }^{3}$, Yasukazu Nakamura ${ }^{3}$, Hoe-Han Goh $^{1 *}$

${ }^{1}$ Institute of Systems Biology, Universiti Kebangsaan Malaysia, 43600 Bangi, Selangor, Malaysia.

${ }^{2}$ Biotechnology Research Institute, Universiti Malaysia Sabah, 88400 Kota Kinabalu, Sabah, Malaysia.

${ }^{3}$ National Institute of Genetics, Mishima, 411-8540 Shizuoka, Japan.

\section{*Correspondence:}

E-mail: gohhh@ukm.edu.my

Short Title: Mitogenome of mangosteen variety Mesta 


\begin{abstract}
Fruits of Garcinia mangostana L. (mangosteen) are rich in nutrients with xanthones found in the pericarp having great pharmaceutical potential. Mangosteen variety Mesta is only found in Malaysia, which tastes sweeter than the common Manggis variety in Southeast Asia. In this study, we report the complete mitogenome of $G$. mangostana L. variety Mesta with a total sequence length of 371,235 bp of which $1.7 \%$ could be of plastid origin. The overall GC content of the mitogenome is $43.8 \%$, comprising 29 protein-coding genes, 3 rRNA genes, and 21 tRNA genes. Repeat and tandem repeat sequences accounted for $5.8 \%$ and $0.15 \%$ of the Mesta mitogenome, respectively. There are 333 predicted RNA-editing sites in Mesta mitogenome. These include the RNA-editing events that generated the start codon of nadl gene and the stop codon of $c c m F C$ gene. Phylogenomic analysis with a maximum likelihood method showed that the mitogenome of mangosteen variety Mesta was grouped under Malpighiales order. This is the first complete mitogenome from the Garcinia genus for future evolutionary studies.
\end{abstract}

Keywords: Garcinia mangostana; mitogenome; SMRT PacBio; structural variants; RNA editing 


\section{Introduction}

Mitochondria are the main organelle of energy production for cell sustainability. Besides chloroplast genomes (plastomes), mitochondrial genomes (mitogenomes) serve as important genetic information for phylogenetic and evolutionary studies ${ }^{1}$. The first land plant mitogenome sequenced was Marchantia polymorpha ${ }^{2}$. Mitogenome sizes are more variable, which range from $66 \mathrm{~kb}^{3}$ to $5.5 \mathrm{Mb}^{4}$ compared to plastomes that are more conserved with the length range from $110 \mathrm{~kb}$ to $200 \mathrm{~kb}^{5}$. Plant mitogenomes are complex due to the rearrangement, duplication, recombination, and horizontal gene transfer between nucleus and organelles (plastids and mitochondria) $)^{6-8}$.

With the advancement of third-generation sequencing technology such as Pacific Biosciences (PacBio) and Oxford Nanopore Technologies (ONT) which can sequence over $100 \mathrm{~kb}$ per molecule and up to 1 million bp, long-read sequencing is becoming more commonly applied in mitogenome assembly ${ }^{9,10}$. The repetitive regions and rearrangement events that are common in mitogenomes hinder assembly with short-read sequencing ${ }^{11}$.

Mangosteen (Garcinia mangostana L.) is well-known as the "Queen of fruits" with sweet and juicy fruit pulp. It is a tropical fruit under the Clusiaceae family ${ }^{12}$ that can be found in Southeast Asia countries ${ }^{13}$. In Malaysia, there is a unique variety Mesta, which is characterized by an oblong shape, thicker mesocarp, and relatively fewer and smaller seeds compared to the common Manggis variety. Despite eight published chloroplast genomes from Garcinia species, there is no mitogenome from Garcinia species reported to date.

In this study, we assembled a complete mitogenome of Mesta using PacBio data ${ }^{14}$ and polished using Illumina short reads ${ }^{15}$. We also compared its structure and gene contents with five closely related species from the same order of Malpighiales and another two species from the Brassicales order, namely Arabidopsis thaliana and Carica papaya (reference used during assembly). The study provides a reference for future evolutionary studies of the Garcinia genus.

\section{Results}

\section{General features of Garcinia mangostana var. Mesta mitogenome}

De novo assembly using Organelle_PBA generated two mitogenome contigs with the length of 389,277 bp (scf7180000000010) and 20,340 bp (scf7180000000011), respectively. The smaller contig was the subset of the larger (master) contig (Supplementary Figure S1). For the 
larger contig that was circular, manual curation was done by removing one of the identical ends $(\sim 18 \mathrm{~kb})$ and a total of 63 bases were added based on the detected variants when short reads were aligned to the trimmed mitogenome (Supplementary Figure S2). The final complete mitogenome of Garcinia mangostana var. Mesta was 371,235 bp which was slightly larger than Arabidopsis thaliana mitogenome (367,808 bp) but smaller than Carica papaya mitogenome (476,890 bp) (Table 1). The average Mesta mitogenome coverage was $129 \times$ using PacBio subreads (Supplementary Figure S3). The mitogenome comprising 29 protein-coding genes, 3 rRNA genes (rrn5, rrn18, and rrn26), and 21 tRNA genes (Figure $1 \&$ Table 2). The total length of protein-coding genes was $28,113 \mathrm{bp}$, which accounted for $7.6 \%$ of the mitogenome. There were only five ribosomal proteins ( $r p l 5, r p l 10, r p l 16, r p s 3$, and $r p s 4$ ) found in the Mesta mitogenome.

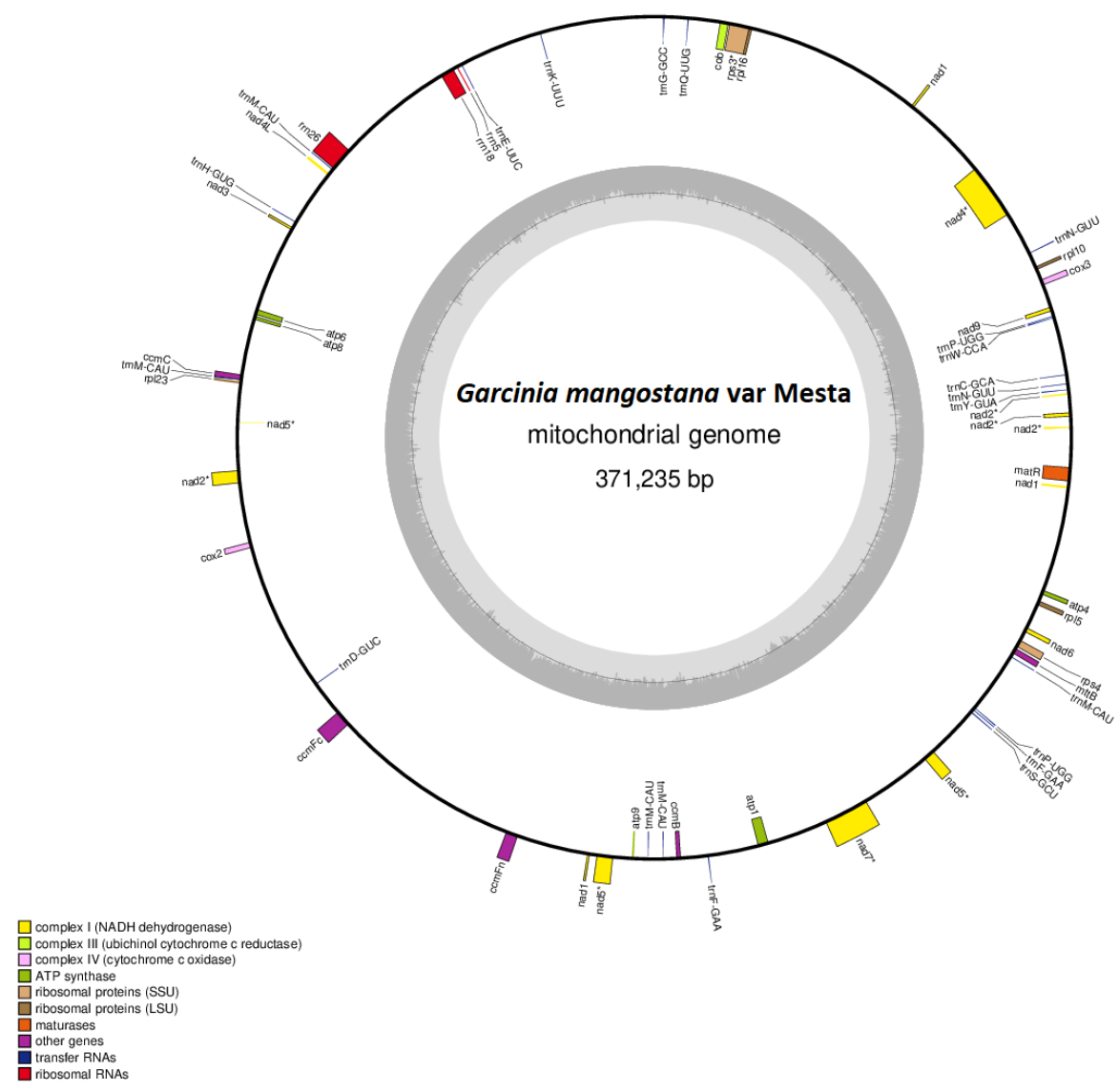

Figure 1. The circular mitochondrial genome of G. mangostana variety Mesta. Genes inside the circle are transcribed clockwise while genes outside the circle are transcribed anticlockwise. The gray bars inside the circle represent the GC content of the sequence. Asterisks $(*)$ indicate genes containing intron(s). 
Table 1. Comparison of the gene content in mitogenomes of different species.

\begin{tabular}{|c|c|c|c|c|c|c|c|c|c|}
\hline Order & Family & Features & $\begin{array}{l}\text { Accession } \\
\text { number }\end{array}$ & $\begin{array}{l}\text { Size } \\
\text { (bp) }\end{array}$ & $\begin{array}{l}\text { GC content } \\
(\%)\end{array}$ & $\begin{array}{l}\text { Number } \\
\text { of gene }\end{array}$ & $\begin{array}{l}\text { Protein- } \\
\text { coding }\end{array}$ & rRNA & tRNA \\
\hline \multirow[t]{2}{*}{ Brassicales } & Brassicaceae & A. thaliana & BK010421 & 367,808 & 44.8 & 58 & 33 & 3 & 22 \\
\hline & Caricaceae & C. papaya & NC_012116.1 & 476,890 & 45.1 & 61 & 39 & 3 & 19 \\
\hline \multirow[t]{6}{*}{ Malpighiales } & Clusiaceae & G. mangostana & OM759996 & 371,235 & 43.8 & 55 & 29 & 3 & 21 \\
\hline & Euphorbiaceae & R. communis & NC_015141.1 & 502,773 & 45.0 & 60 & 37 & 3 & 20 \\
\hline & Salicaceae & P. alba & NC_041085.1 & 838,420 & 44.8 & 58 & 33 & 3 & 22 \\
\hline & & P. davidiana & NC_035157.1 & 779,361 & 44.8 & 58 & 33 & 3 & 22 \\
\hline & & P. tremula & NC_028096.1 & 783,442 & 44.7 & 58 & 33 & 3 & 22 \\
\hline & Passifloraceae & P. edulis & NC_050950.1 & 680,480 & 44.7 & 73 & 41 & 3 & 29 \\
\hline
\end{tabular}


Table 2. List of genes in Garcinia mangostana var. Mesta mitogenome.

\begin{tabular}{|c|c|}
\hline Group & Gene \\
\hline Complex I & nad1, nad2, nad3, nad4, nad4L, nad5, nad6, nad7, nad9 \\
\hline Complex III & $c o b$ \\
\hline Complex IV & $\operatorname{cox} 1, \operatorname{cox} 2, \operatorname{cox} 3$ \\
\hline Complex V & atpl, atp4, atp6, atp8, atp9 \\
\hline Cytochrome c biogenesis & $c c m B, c c m C, c c m F c, c c m F n$ \\
\hline Ribosome large subunit & rpl5, rpl10, rpl16 \\
\hline Ribosome small subunit & rps3, rps4, \\
\hline Intron maturase & matR \\
\hline SecY-independent transporter & $m t t B$ \\
\hline rRNA genes & $r r n 5, r r n L, r r n S$ \\
\hline tRNA genes & $\begin{array}{llll}\operatorname{trn} C-G C A, & \operatorname{trn} D-G U C, \operatorname{trn} E-U U C, & \operatorname{trn} F-G A A & (\mathrm{X} 2), \\
\operatorname{trn} G-G C C, & \operatorname{trn} H-G U G, \operatorname{trn} K-U U U, & \operatorname{trn} M-C A U & (\mathrm{X} 5), \\
\operatorname{trn} N-G U U & (\mathrm{X} 2), \operatorname{trn} P-U G G(\mathrm{X} 2), & \operatorname{trn} Q-U U G, & \operatorname{trn} S- \\
G C U, \text { trnW-CCA, trnY-GUA } & \end{array}$ \\
\hline
\end{tabular}

\section{Comparison of Mitogenome Gene content of different species}

Comparison of the mitogenomes gene content of different species (Supplementary Table S1) showed that all the mitogenomes encoded the basic core set of 24 protein-coding genes. However, the mitogenome of Passiflora edulis encoded two copies of the genes $c c m B$, nad $4 L$, $n a d 6$, and nad7 and four copies of the gene cox2. Mitogenome of Arabidopsis thaliana also encoded two copies of atp6 gene with the length of $1158 \mathrm{bp}$ and $1050 \mathrm{bp}$, respectively. Two copies of $c c m F N$ gene were also found in the mitogenome of Carica papaya. The nadl gene in Mesta was found only consisted of 3 exons instead of 5 as observed in other species despite the total length of its CDS sequence being almost like the other species. Most of the ribosomal proteins were found missing in both Salicaceae and Clusiaceae families from the order Malpighiales. Noticeably, gene rps12 was missing from Mesta.

\section{Distribution of tRNAs}

The 21 tRNAs identified in Mesta mitogenome only code for 14 amino acids (Ser, Phe, Asn, Met, Pro, Gly, Lys, Gln, Tyr, His, Trp, Asp, Glu, Cys). Two out of 21 tRNAs had a chloroplastorigin while the rest were mitochondrial-origin. However, the tRNA genes code for the other six amino acids (Leu, Ile, Thr, Ala, Val, Arg) were not detected. Among the 21 tRNAs, one of them was predicted to have one intron (Supplementary Table S2).

\section{Plastome-derived sequences}


There were five plastome-derived sequences with an identity of more than $80 \%$ and a sequence length of at least $100 \mathrm{bp}$ (Table 3 ) found in the mitogenome. This accounted for a total length of $6,214 \mathrm{bp}$ which was $1.7 \%$ of the mitogenome. The plastome genes contained were $r p l 2$ (partial), rpl23, trnl-CAU, ndhA (partial), ndhH, rps15 (partial), atpE (partial), atpB, rps3 (partial), and trnD-GUC. Three of the fragments were found at the plastome large single-copy (LSC) region and one from the single-copy (SSC) region and inverted repeat (IR) regions, respectively.

Table 3. Plastid gene insertions in the mitochondrial genome of Mesta.

\begin{tabular}{lclll}
\hline Plastid gene & Length (bp) & Position & Identity (\%) & Location \\
\hline$r p l 2^{\wedge}, r p l 23, t r n l-C A U$ & 1,418 & $178,888-177,471$ & 97.77 & IR \\
$n d h A^{\wedge}, n d h H, r p s 15^{\wedge}$ & 2,476 & $21,314-18,839$ & 97.68 & SSC \\
$a t p E^{\wedge}, a t p B$ & 1,882 & $72,995-71,114$ & 97.54 & LSC \\
$r p s 3^{\wedge}$ & 289 & $310,031-310,319$ & 87.06 & LSC \\
$t r n D-G U C$ & 149 & $222,409-222,557$ & 85.81 & LSC \\
\hline partial & & & &
\end{tabular}

\section{Introns and RNA Editing}

There were 20 introns distributed among 8 protein-coding genes (ccmFC, coxl, nad1, nad2, nad4, nad5, nad7, and rps3) in Mesta mitogenome. Among them, nad1, nad2, and nad5 genes were trans-spliced. The maximum number of introns found in a gene were four which can be found at certain nad gene families such as nad2, nad5, and nad7.

A total of 333 putative RNA-editing sites had been predicted using PREP-MT (Supplementary Table S4). Among the CDS, gene nad4 contained the highest number of predicted editing sites (35) while there was no editing site found at the atp9 gene. A total of three annotated genes did not start with the start codon ATG (atp6, nadl, and rpl16) (Supplementary Table S3). Among them, the ACG site at the beginning sequence of nadl was putatively predicted to be one of the editing sites that converted it into the ATG start codon. Similarly, early termination was predicted at the gene $c c m F C$ sequence which converted the CGA into the stop codon TGA.

\section{Repeat sequences of Mesta mitochondrial DNA}

A total of 64 repeat sequences comprising the forward and palindromic repeats were detected. The repeat sequences ranged from $51 \mathrm{bp}$ to $4,172 \mathrm{bp}$ with a total size of $21,356 \mathrm{bp}$ which accounted for $5.8 \%$ of the total Mesta mitogenome (Supplementary Table S5). There were 
three forward repeat sequences with lengths of 4,172 bp, 2,031 bp, and 1,641 bp. As for the tandem repeats, it constituted $0.15 \%$ of the mitogenome (Supplementary Table S6).

\section{Phylogenomic Analysis}

A total of 22 (excluding $c c m F n$ and $m t t B$ ) out of 24 basic core sets of protein-coding genes were used for phylogenomic analysis, which separated the 15 species into different groups based on the order. Mesta was grouped under Malpighiaes together with P. edulis, S. suchowensis, S. purpurea, P. alba, P. tremula, and P. davidiana.

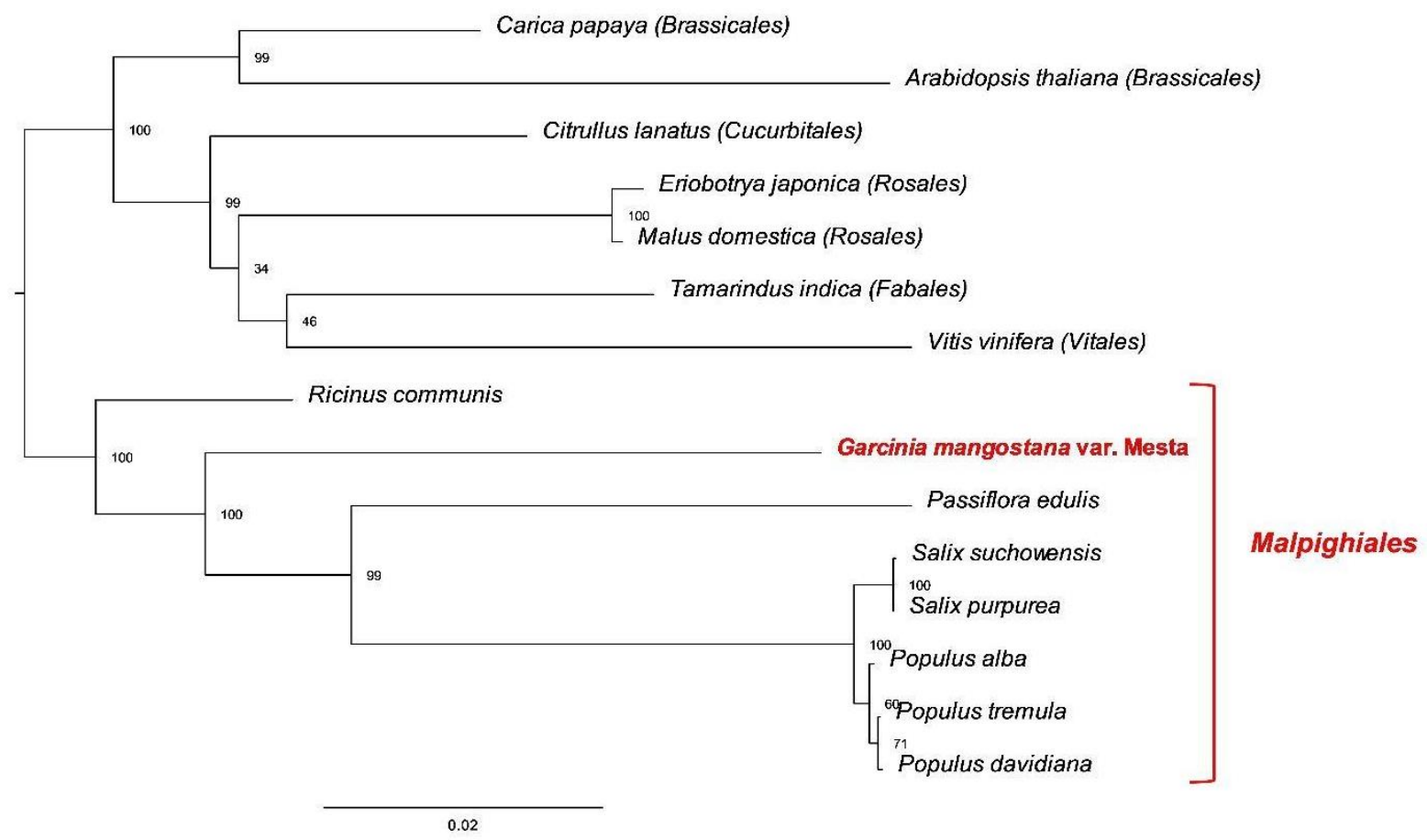

Figure 2. Phylogenomic tree (maximum likelihood) of 15 species based on 22 protein-coding genes. The parentheses and red line indicate the order name.

\section{Discussion}

Due to the high complexity of plant mitogenome with large repetitive regions, long-read sequencing is superior in mitogenome assembly ${ }^{4,16}$. In this study, a total of two Mesta mitogenome contigs were obtained using PacBio data. The shorter contig was a subset of the longer one with the size of 371,235 bp (after manual curation) and was considered as the complete Mesta mitogenome. It is not uncommon to have multiple mitogenome contigs in plants to exist in both circular and linear structures due to intramolecular recombination events $^{11,17,18}$. For instance, there were 10 contigs in Fagopyrum esculentum ${ }^{19}$ and 13 contigs in Picea sitchensis ${ }^{4}$. 
Mesta mitogenome encoded the basic core set of 24 protein-coding genes ${ }^{20}$ that include genes encoding proteins of the electron transport chain (complex I, III, IV, V, and cytoplasmic membrane proteins) commonly found in plant mitogenome ${ }^{21}$. However, Mesta mitogenome size was relatively small compared to other plant mitogenomes ${ }^{22}$ from the same order, Malpighiales (Table 1), due to the reduced number of ribosomal proteins and missing genes encoding respiratory chain complex II, sdh3 and sdh4 (Supplementary Table S3). These protein-coding genes could be lost during evolution and might be transferred to the nuclear genome as observed in other angiosperm mitogenomes ${ }^{23-25}$ such as S. latifolia ${ }^{26}$, S. noctiflora ${ }^{27}$, P. dactylifera, and A. indica ${ }^{21,25}$. For instance, rps 12 was not found in Mesta mitogenome as well as Oenothera and Zostera marina ${ }^{28,29}$.

A complete set of tRNAs coding for 20 amino acids is required for protein translation in plant mitogenomes. However, currently, there was no complete set of tRNA genes found in the mitogenome of angiosperm ${ }^{30}$. For the Mesta mitogenome, a total of six amino acids encoded by tRNAs (Leu, Ile, Thr, Ala, Val, Arg) were not detected and these tRNAs types were generally reported missing in angiosperm mitogenomes ${ }^{30}$. In the mitogenome of $S$. latifolia, the majority of the tRNAs were reported lost with only nine types of amino acids ${ }^{26}$. The loss of tRNAs can be either replaced by tRNAs from the chloroplast or nuclear genome ${ }^{26,31}$.

There are several factors attributed to the differences of plant mitogenome lengths, including the integration of nuclear and plastid genomes as well as the number and length of non-coding regions ${ }^{20}$. Chloroplast sequences can be found in plant mitogenomes as there were integration events during evolution. The integration of plastome sequences into mitogenomes can range from 1 to $12 \%{ }^{32}$. For the Mesta mitogenome, the integration rate was $1.7 \%$ which was smaller compared to other plants such as watermelon $(7.6 \%)^{33}$, P. dactylifera $(10.3 \%)^{21}$, and $C$. pepo $(11.6 \%)^{34}$.

The repetitive regions found in the intergenic regions of mitogenome were in variable types such as short repeats, tandem repeats, and long complex repeats ${ }^{21,22,35,36}$. The large repeats $(>1 \mathrm{~kb})$ might cause homologous recombination and eventually lead to the different configuration of the mitogenomes ${ }^{37}$. Apart from large repeats, both direct and inverse repeats also contribute to the subgenomic molecules ${ }^{7}$. Repeats detected in Mesta mitogenome using web-based REPuter was low (5.8\%) compared to C. melo $(42.7 \%)^{38}$, V. vinifera $(6.8 \%)^{39}$, and $N$. colorata $(48.89 \%)^{40}$ but higher than $P$. dactylifera $(2.3 \%)^{21}$. Similarly, the tandem repeats detected in Mesta mitogenome were also low which was $0.15 \%$ compared to $0.33 \%$ in $P$. dactylifera ${ }^{21}$. 
RNA-editing events are essential in plant development and stress response ${ }^{41}$. The most common RNA editing events in plant organelles (mitochondria and plastids) were the conversion of C-to- $\mathrm{U}^{42}$. RNA editing can lead to the start codon/stop codon generation, eliminate premature stop codon, change the splicing site, affect the RNA structure, and cause instability of $\mathrm{RNAs}^{41}$. It is predicted that RNA-editing events generated the start codon in nadl and the stop codon in $c c m F C$ genes of Mesta mitogenome. The start codon of nadl gene in several species such as $A$. alpina $^{43}, B$. stricta ${ }^{44}$, and C. rubella ${ }^{45}$ was also formed by RNA editing. On the other hand, stop codon prediction in $c c m F C$ gene sequence had also been reported in A. thaliana and C. bursa-pastoris ${ }^{1}$. The GTG in rpll6 might be a translation start codon as similar observations were found in maize, Marchantia, and Petunia mitogenomes ${ }^{46}$.

\section{Conclusion}

The complete mitogenome of Mesta was successfully assembled. The Mesta mitogenome length was relatively smaller than other species in the same order due to the loss of the majority of ribosomal proteins and both $s d h$ genes. Phylogenetic analysis based on the 22 protein-coding genes among the 15 selected species showed that Mesta was clustered within the Malpighiales order. The mitogenome can serve as a good reference to study the regulation of the mitogenome genes.

\section{Materials and Methods}

\section{Mitochondrial Genome Assembly}

Genome sequences of Mesta variety were obtained from the NCBI SRA database with the accession numbers SRX2718652 to SRX2718659 for PacBio long-read data $(9.5 \mathrm{~Gb})^{14}$ and SRX270978 for Illumina short reads $(50.2 \mathrm{~Gb})^{15}$. CANU v2.0 ${ }^{47}$ was used to perform PacBio raw data correction and trimming using default parameters. Next, non-mitogenome reads were removed by sequence alignment of each read against a Carica papaya mitogenome (accession no. NC_012116.1) which was used as the reference genome. Then, de novo assembly was performed using Organelle_PBA software ${ }^{48}$. Manual curation was performed to obtain the complete Mesta mitogenome.

\section{Genome annotation}

Gene annotation was conducted using both GeSeq ${ }^{49}$ and MITOFY web server ${ }^{34}$. Web-based tRNA-scan v2.0 server (http://lowelab.ucsc.edu/tRNAscan-SE/index.html ${ }^{50}$ was used to 
annotate tRNA genes. The physical mitogenome map was generated using Organellar Genome DRAW (OGDRAW v1.3.1) program with default parameters ${ }^{51}$.

\section{Identification of plastome derived sequences}

Plastome-derived sequences were identified by aligning plastome (accession number: MZ823408) and mitogenome of G. mangostana var. Mesta (accession number: OM759996) $\begin{array}{llll}\text { using } & \text { NCBI-Nucleotide } & \text { BLAST } & \text { (BLASTN) }\end{array}$ (https://blast.ncbi.nlm.nih.gov/Blast.cgi) with at least $80 \%$ sequence identity and alignment length greater than $100 \mathrm{bp}$.

\section{Analysis of RNA-Editing and Substitution Rate}

Putative RNA-editing sites in protein-coding genes of Mesta mitogenome were predicted using PREP-mt web-based program (http://prep.unl.edu//) ${ }^{52}$. The cut-off value was set at 0.6 to obtain an accurate prediction.

\section{Analysis of Repetitive Sequences}

The repeat sequences were identified using web-based REPuter (https://bibiserv.cebitec.unibielefeld.de/reputer// ${ }^{53}$ with a minimum length of repeat size of $50 \mathrm{bp}$. Web-based tool Tandem Repeats Finder version $4.09^{54}$ (https://tandem.bu.edu/trf/trf.basic.submit.html) was used to identify tandem repeats in Mesta mt using default parameter.

\section{Phylogenomic Analysis}

To examine the evolutionary relationship of G. mangostana var. Mesta, a maximum likelihood (ML) phylogenomic tree was inferred based on 22 protein-coding genes (Figure 1). The amino acids sequences of these 22 protein-coding genes were concatenated and aligned using MAFFT version 7 online tool (https://mafft.cbrc.jp/alignment/server/) ${ }^{55}$. The maximum likelihood (ML) analysis was performed using RAxML-NG v1.0.2 tool $^{56}$ based on the selected model STMTREV+I+G4+F in the ModelTest-NG v0.1.6 ${ }^{57}$.

\section{Data Availability Statement}

The complete mitogenome sequence of Garcinia mangostana var. Mesta has been submitted to GenBank with the accession number OM759996. 


\section{References}

1 O'Conner, S. \& Li, L. Mitochondrial Fostering: The Mitochondrial Genome May Play a Role in Plant Orphan Gene Evolution. Frontiers in Plant Science 11, doi:10.3389/fpls.2020.600117 (2020).

2 Oda, K. et al. Gene organization deduced from the complete sequence of liverwort Marchantia polymorpha mitochondrial DNA: a primitive form of plant mitochondrial genome. Journal of molecular biology 223, 1-7 (1992).

3 Skippington, E., Barkman, T. J., Rice, D. W. \& Palmer, J. D. Miniaturized mitogenome of the parasitic plant Viscum scurruloideum is extremely divergent and dynamic and has lost all nad genes. Proceedings of the National Academy of Sciences 112, E3515-E3524 (2015).

4 Jackman, S. D. et al. Complete mitochondrial genome of a gymnosperm, Sitka spruce (Picea sitchensis), indicates a complex physical structure. Genome biology and evolution 12, 1174-1179 (2020).

5 Sugiura, M. The chloroplast genome. Essays in biochemistry 30, $49-57$ (1995).

6 Bergthorsson, U., Richardson, A. O., Young, G. J., Goertzen, L. R. \& Palmer, J. D. Massive horizontal transfer of mitochondrial genes from diverse land plant donors to the basal angiosperm Amborella. Proceedings of the National Academy of Sciences 101, 17747-17752 (2004).

7 Møller, I. M., Rasmusson, A. G. \& Van Aken, O. Plant mitochondria-past, present and future. The Plant Journal (2021).

8 Sanchez-Puerta, M. V. et al. Genome-scale transfer of mitochondrial DNA from legume hosts to the holoparasite Lophophytum mirabile (Balanophoraceae). Molecular phylogenetics and evolution 132, 243-250 (2019).

9 Midha, M. K., Wu, M. \& Chiu, K.-P. Long-read sequencing in deciphering human genetics to a greater depth. Human genetics 138, 1201-1215 (2019).

10 Xiao, T. \& Zhou, W. The third generation sequencing: the advanced approach to genetic diseases. Transl Pediatr 9, 163-173, doi:10.21037/tp.2020.03.06 (2020).

11 Sloan, D. B. One ring to rule them all? Genome sequencing provides new insights into the 'master circle'model of plant mitochondrial DNA structure. New Phytologist 200, 978-985 (2013).

12 Nazre, M., Newman, M., Pennington, R. \& Middleton, D. Taxonomic revision of Garcinia section Garcinia (Clusiaceae). Phytotaxa 373, 1-52 (2018).

13 Aizat, W. M., Jamil, I. N., Ahmad-Hashim, F. H. \& Noor, N. M. Recent updates on metabolite composition and medicinal benefits of mangosteen plant. PeerJ 7, e6324, doi:10.7717/peerj.6324 (2019).

14 Midin, M. R. et al. SMRT sequencing data for Garcinia mangostana L. variety Mesta. Genomics Data 12, 134-135, doi:10.1016/j.gdata.2017.04.003 (2017).

15 Abu Bakar, S., Kumar, S., Loke, K. K., Goh, H. H. \& Mohd Noor, N. DNA shotgun sequencing analysis of Garcinia mangostana L. variety Mesta. Genomics Data 12, 118-119, doi:10.1016/j.gdata.2017.05.001 (2017).

16 Omelchenko, D. O. et al. Assembly and Analysis of the Complete Mitochondrial Genome of Capsella bursa-pastoris. Plants 9, 469 (2020).

17 Kozik, A. et al. The alternative reality of plant mitochondrial DNA: One ring does not rule them all. PLoS genetics 15, e1008373 (2019).

18 Mower, J. P., Case, A. L., Floro, E. R. \& Willis, J. H. Evidence against equimolarity of large repeat arrangements and a predominant master circle structure of the 
bioRxiv preprint doi: https://doi.org/10.1101/2022.02.23.481586; this version posted February 25, 2022. The copyright holder for this preprint (which was not certified by peer review) is the author/funder, who has granted bioRxiv a license to display the preprint in perpetuity. It is made available under aCC-BY-NC-ND 4.0 International license.

mitochondrial genome from a monkeyflower (Mimulus guttatus) lineage with cryptic CMS. Genome Biol Evol 4, 670-686, doi:10.1093/gbe/evs042 (2012).

19 Logacheva, M. D., Schelkunov, M. I., Fesenko, A. N., Kasianov, A. S. \& Penin, A. A. Mitochondrial genome of Fagopyrum esculentum and the genetic diversity of extranuclear genomes in buckwheat. Plants 9, 618 (2020).

20 Zardoya, R. Recent advances in understanding mitochondrial genome diversity. F1000Research 9 (2020).

21 Fang, Y. et al. A complete sequence and transcriptomic analyses of date palm (Phoenix dactylifera L.) mitochondrial genome. PloS one 7, e37164 (2012).

22 Wynn, E. L. \& Christensen, A. C. Repeats of unusual size in plant mitochondrial genomes: identification, incidence and evolution. G3: Genes, Genomes, Genetics 9 , 549-559 (2019).

23 Adams, K. L., Qiu, Y.-L., Stoutemyer, M. \& Palmer, J. D. Punctuated evolution of mitochondrial gene content: high and variable rates of mitochondrial gene loss and transfer to the nucleus during angiosperm evolution. Proceedings of the National Academy of Sciences 99, 9905-9912 (2002).

24 Asaf, S. et al. Mitochondrial genome analysis of wild rice (Oryza minuta) and its comparison with other related species. PloS one 11, e0152937 (2016).

25 Choi, K.-S. \& Park, S. Complete Plastid and Mitochondrial Genomes of Aeginetia indica Reveal Intracellular Gene Transfer (IGT), Horizontal Gene Transfer (HGT), and Cytoplasmic Male Sterility (CMS). International Journal of Molecular Sciences 22, 6143 (2021).

26 Sloan, D. B., Alverson, A. J., Štorchová, H., Palmer, J. D. \& Taylor, D. R. Extensive loss of translational genes in the structurally dynamic mitochondrial genome of the angiosperm Silene latifolia. BMC Evolutionary Biology 10, 1-15 (2010).

27 Sloan, D. B., MacQueen, A. H., Alverson, A. J., Palmer, J. D. \& Taylor, D. R. Extensive Loss of RNA Editing Sites in Rapidly Evolving Silene Mitochondrial Genomes: Selection vs. Retroprocessing as the Driving Force. Genetics 185, 13691380, doi:10.1534/genetics.110.118000 (2010).

28 Grohmann, L., Brennicke, A. \& Schuster, W. The mitochondrial gene encoding ribosomal protein $\mathrm{S} 12$ has been translocated to the nuclear genome in Oenothera. Nucleic acids research 20, 5641-5646 (1992).

29 Petersen, G. et al. Mitochondrial genome evolution in Alismatales: Size reduction and extensive loss of ribosomal protein genes. PLoS One 12, e0177606 (2017).

30 Michaud, M., Cognat, V., Duchêne, A. M. \& Maréchal-Drouard, L. A global picture of tRNA genes in plant genomes. The Plant Journal 66, 80-93 (2011).

31 Dietrich, A., Small, I., Cosset, A., Weil, J. \& Maréchal-Drouard, L. Editing and import: strategies for providing plant mitochondria with a complete set of functional transfer RNAs. Biochimie 78, 518-529 (1996).

32 Mower, J. P., Sloan, D. B. \& Alverson, A. J. Plant mitochondrial genome diversity: the genomics revolution. Plant Genome Diversity Volume 1, 123-144 (2012).

33 Cui, H. et al. Comparative analysis of nuclear, chloroplast, and mitochondrial genomes of watermelon and melon provides evidence of gene transfer. Scientific reports 11, 1-9 (2021).

34 Alverson, A. J. et al. Insights into the Evolution of Mitochondrial Genome Size from Complete Sequences of Citrullus lanatus and Cucurbita pepo (Cucurbitaceae). Molecular Biology and Evolution 27, 1436-1448, doi:10.1093/molbev/msq029 (2010). 
bioRxiv preprint doi: https://doi.org/10.1101/2022.02.23.481586; this version posted February 25, 2022. The copyright holder for this preprint (which was not certified by peer review) is the author/funder, who has granted bioRxiv a license to display the preprint in perpetuity. It is made available under aCC-BY-NC-ND 4.0 International license.

35 Alverson, A. J., Zhuo, S., Rice, D. W., Sloan, D. B. \& Palmer, J. D. The mitochondrial genome of the legume Vigna radiata and the analysis of recombination across short mitochondrial repeats. PLoS one 6, e16404 (2011).

36 Figueroa-Martinez, F., Nedelcu, A. M., Smith, D. R. \& Reyes-Prieto, A. The plastid genome of Polytoma uvella is the largest known among colorless algae and plants and reflects contrasting evolutionary paths to nonphotosynthetic lifestyles. Plant physiology 173, 932-943 (2017).

37 Gualberto, J. M. et al. The plant mitochondrial genome: dynamics and maintenance. Biochimie 100, 107-120 (2014).

38 Rodríguez-Moreno, L. et al. Determination of the melon chloroplast and mitochondrial genome sequences reveals that the largest reported mitochondrial genome in plants contains a significant amount of DNA having a nuclear origin. $B M C$ genomics 12, 1-14 (2011).

39 Goremykin, V. V., Salamini, F., Velasco, R. \& Viola, R. Mitochondrial DNA of Vitis vinifera and the issue of rampant horizontal gene transfer. Molecular biology and evolution 26, 99-110 (2009).

40 Dong, S. et al. The complete mitochondrial genome of the early flowering plant Nymphaea colorata is highly repetitive with low recombination. BMC Genomics 19, 614, doi:10.1186/s12864-018-4991-4 (2018).

41 Tang, W. \& Luo, C. Molecular and functional diversity of RNA editing in plant mitochondria. Molecular biotechnology 60, 935-945 (2018).

42 Hao, W. et al. RNA Editing and Its Roles in Plant Organelles. Frontiers in Genetics, 1747 (2021).

$43 \mathrm{Xu}, \mathrm{Y} . \& \mathrm{Bi}, \mathrm{C}$. The complete mitochondrial genome sequence of an alpine plant Arabis alpina. Mitochondrial DNA Part B 3, 725-727 (2018).

$44 \mathrm{Li}, \mathrm{J} ., \mathrm{Bi}, \mathrm{C}$., Tu, J. \& Lu, Z. The complete mitochondrial genome sequence of Boechera stricta. Mitochondrial DNA Part B 3, 896-897 (2018).

45 Lin, H. \& Bai, D. The complete mitochondrial genome of a highly selfing species Capsella rubella. Mitochondrial DNA Part B 4, 1907-1908 (2019).

46 Bock, H., Brennicke, A. \& Schuster, W. Rps3 and rpll6 genes do not overlap in Oenothera mitochondria: GTG as a potential translation initiation codon in plant mitochondria? Plant Mol Biol 24, 811-818, doi:10.1007/bf00029863 (1994).

47 Walker, B. J. et al. Pilon: An Integrated Tool for Comprehensive Microbial Variant Detection and Genome Assembly Improvement. PLOS ONE 9, e112963, doi:10.1371/journal.pone.0112963 (2014).

48 Soorni, A., Haak, D., Zaitlin, D. \& Bombarely, A. Organelle_PBA, a pipeline for assembling chloroplast and mitochondrial genomes from PacBio DNA sequencing data. BMC genomics 18, 49-49, doi:10.1186/s12864-016-3412-9 (2017).

49 Tillich, M. et al. GeSeq - versatile and accurate annotation of organelle genomes. Nucleic Acids Res 45, W6-w11, doi:10.1093/nar/gkx391 (2017).

50 Chan, P. P. \& Lowe, T. M. in Gene prediction 1-14 (Springer, 2019).

51 Greiner, S., Lehwark, P. \& Bock, R. OrganellarGenomeDRAW (OGDRAW) version 1.3.1: expanded toolkit for the graphical visualization of organellar genomes. Nucleic Acids Research 47, W59-W64, doi:10.1093/nar/gkz238 (2019).

52 Mower, J. P. The PREP suite: predictive RNA editors for plant mitochondrial genes, chloroplast genes and user-defined alignments. Nucleic Acids Research 37, W253W259, doi:10.1093/nar/gkp337 (2009).

53 Kurtz, S. et al. REPuter: the manifold applications of repeat analysis on a genomic scale. Nucleic acids research 29, 4633-4642, doi:10.1093/nar/29.22.4633 (2001). 
54 Benson, G. Tandem repeats finder: a program to analyze DNA sequences. Nucleic Acids Res 27, 573-580, doi:10.1093/nar/27.2.573 (1999).

55 Katoh, K., Rozewicki, J. \& Yamada, K. D. MAFFT online service: multiple sequence alignment, interactive sequence choice and visualization. Briefings in bioinformatics 20, 1160-1166 (2019).

56 Kozlov, A. M., Darriba, D., Flouri, T., Morel, B. \& Stamatakis, A. RAxML-NG: a fast, scalable and user-friendly tool for maximum likelihood phylogenetic inference. Bioinformatics 35, 4453-4455, doi:10.1093/bioinformatics/btz305 (2019).

57 Darriba, D. et al. ModelTest-NG: A New and Scalable Tool for the Selection of DNA and Protein Evolutionary Models. Molecular Biology and Evolution 37, 291-294, doi:10.1093/molbev/msz189 (2020).

\section{Acknowledgments}

We would like to acknowledge the support of this research by Universiti Kebangsaan Malaysia (UKM) Research University grant AP-2012-018. The group is currently supported by UKM Research University grant DIP-2020-005 (H-HG) and NIG-JOINT grant 2021 (2A2021) (YN and H-HG), Japan.

\section{Author Contribution Statement}

C.C.W. and H.H.G. conceived and planned the experiment. C.C.W. wrote the paper and analyzed the data. Y.N. contributed to the data analysis. H.H.G., N.A.N.M, V.K.S., M.A., and Y.N. reviewed and edited the manuscript. All authors read and approved the manuscript.

\section{Additional information}

Supplementary Tables: Figure S1-S3

Supplementary Figures: Table S1-S6

\section{Conflict of Interest}

The authors declare no competing interests.

\section{Legends}

\section{Figures}

Figure 1. The circular mitochondrial genome of G. mangostana variety Mesta. Genes inside the circle are transcribed clockwise while genes outside the circle are transcribed anticlockwise as indicated by the gray arrows. The gray bars inside the circle represent the GC content of the sequence. Asterisks (*) indicate genes containing intron(s). 
Figure 2. Phylogenomic tree (maximum likelihood) of 15 species based on 22 protein-coding genes. The parentheses and red line indicate the order name.

\section{Tables}

Table 1. Comparison of the gene content in mitogenomes of different species.

Table 2. List of genes in Garcinia mangostana var. Mesta mitogenome.

Table 3. Plastid gene insertions in the mitochondrial genome of Mesta. 